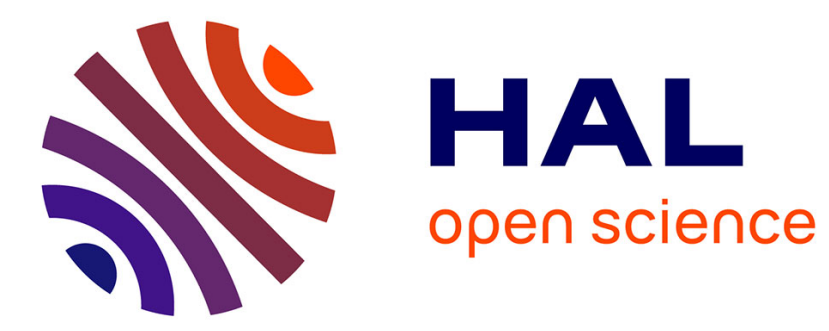

\title{
EXPRESSIONS SIMPLIFIÉES DU FLUX D'ÉNERGIE DANS LES PLAQUES
}

Jean-Claude Pascal

\section{To cite this version:}

Jean-Claude Pascal. EXPRESSIONS SIMPLIFIÉES DU FLUX D'ÉNERGIE DANS LES PLAQUES. Journal de Physique IV Proceedings, 1992, 02 (C1), pp.C1-515-C1-518. 10.1051/jp4:19921111 . jpa00251066

\section{HAL Id: jpa-00251066 https://hal.science/jpa-00251066}

Submitted on 1 Jan 1992

HAL is a multi-disciplinary open access archive for the deposit and dissemination of scientific research documents, whether they are published or not. The documents may come from teaching and research institutions in France or abroad, or from public or private research centers.
L'archive ouverte pluridisciplinaire HAL, est destinée au dépôt et à la diffusion de documents scientifiques de niveau recherche, publiés ou non, émanant des établissements d'enseignement et de recherche français ou étrangers, des laboratoires publics ou privés. 


\title{
EXPRESSIONS SIMPLIFIÉES DU FLUX D'ENERGIE DANS LES PLAQUES
}

\author{
J.-C. PASCAL \\ Centre Technique des Industries Mécaniques (CETIM), 52 avenue Félix-Louat, F-60300 Senlis, \\ France
}

\begin{abstract}
SUMMARY - The equations for the energy flux carried by bending waves can be approximated using a thirteen point difference pattern for the spatial derivatives in plates. But this formulation can be simplified by introducing different degrees of approximation, making them accessible to measurement by techniques of finite differences between sensors. A conventional two point method gives acceptable results in comparison with the complete thirteen point method. This paper discusses this surprizing establishment.
\end{abstract}

\section{Introduction}

L'intensité vibratoire des ondes de flexion dans les plaques est décrite par une relation comportant des produits entre différentes dérivées spatiales du champ de vitesse vibratoire normale à la surface de la structure. Pour rendre cette quantité accessible à la mesure, Pavić [1] a tiré de cette expression une formulation complète où les dérivées spatiales sont approchées par des différences finies. La détermination par cette méthode du vecteur dans le plan (les composantes $x$ et $y$ ), conduit à mesurer 34 interspectres indépendants [2] calculés entre les paires choisies parmi les 13 accélérométres nécessaires. A cause de sa complexité de mise en cuvre, cette méthode n'a jamais été expérimentée. Dans une première approche de la mesure du flux en 1969, Noiseux [3] avait établi une formulation approchée basée sur un modèle de propagation dans une plaque infinie. Les hypothèses avancées sont très restrictives puisqu'elles supposent qu'on se trouve en champ lointain et en champ libre, mais le nombre de capteurs se réduit à 4 et seulement deux interspectres doivent être mesurés. C'est cette dernière formulation qui a été employée jusqu'à aujourd'hui dans des travaux expérimentaux $[4,5,6]$, sans qu'on ait pu la justifier dans le cas de structures finies. Des études par simulation numérique ont montré qu'elle conduit à d'importantes erreurs près des sources [2] mais à des résultats souvents acceptables en champ lointain [7], ce qui est assez surprenant en considérant l'hypothèse de champ libre qui a permis de l'obtenir. Dans cet article, nous donnons une formulation exacte pour le champ lointain et nous étudions les différentes approximations possibles.

\section{Modèle de champ lointain}

L'intensité vibratoire des ondes de flexion dans une plaque (ou densité de flux de puissance par unité de largeur $[\mathrm{W} / \mathrm{m}]$ ), est une quantité vectorielle qui peut s'exprimer à partir de la vitesse normale $v[1]$,

$$
\begin{aligned}
& I_{x}=\frac{B}{2 \omega} \operatorname{Im}\left\{\frac{\partial}{\partial x}\left(\nabla^{2} v\right) v^{*}-\left(\frac{\partial^{2} v}{\partial x^{2}}+\nu \frac{\partial^{2} v}{\partial y^{2}}\right) \frac{\partial v^{*}}{\partial x}-(1-\nu) \frac{\partial^{2} v}{\partial x \partial y} \frac{\partial v^{*}}{\partial y}\right\} \\
& I_{y}=\frac{B}{2 \omega} \operatorname{Im}\left\{\frac{\partial}{\partial y}\left(\nabla^{2} v\right) v^{*}-\left(\frac{\partial^{2} v}{\partial y^{2}}+\nu \frac{\partial^{2} v}{\partial x^{2}}\right) \frac{\partial v^{*}}{\partial y}-(1-\nu) \frac{\partial^{2} v}{\partial x \partial y} \frac{\partial v^{*}}{\partial x}\right\} .
\end{aligned}
$$


avec $B=E h^{3} / 12\left(1-\nu^{2}\right)$ le coefficient de raideur à la flexion, où $E$ est le module d'Young, $h$ l'épaisseur de la plaque et $\nu$ le coefficient de Poisson. Pour évaluer l'influence de chacun des termes de l'équation (1) de l'intensité vibratoire des ondes de flexion, on utilise un modèle d'onde basé sur la sommation d'ondes planes se propageant dans des directions différentes

$$
v(x, y)=\sum_{m} v_{m}(x, y)=\sum_{m} V_{m} \exp \left[-j k\left(x \cos \alpha_{m}+y \sin \alpha_{m}\right)\right]
$$

où $k=(\omega)^{1 / 2}\left(M_{s} / B\right)^{1 / 4}$ est le nombre d'onde de flexion dans la plaque $\left(M_{s}\right.$, masse par unité de surface de la plaque). Ce modèle n'est pas apte à représenter le champ proche des sources ou des discontinuités, mais donne une bonne approximation des champs d'interférence créés par les réflexions multiples sur les bords d'une plaque. Pour calculer les termes de l'équation (1), on utilisera

$$
\begin{array}{cc}
\frac{\partial v}{\partial x}=-j k \sum_{m} v_{m} \cos \alpha_{m}, & \frac{\partial^{2} v}{\partial x^{2}}=-k^{2} \sum_{m} v_{m} \cos ^{2} \alpha_{m}, \\
\frac{\partial^{2} v}{\partial x \partial y}=-k^{2} \sum_{m} v_{m} \cos \alpha_{m} \sin \alpha_{m}, & \nabla^{2} v=\frac{\partial^{2} v}{\partial x^{2}}+\frac{\partial^{2} v}{\partial y^{2}}=-k^{2} v .
\end{array}
$$

En fonction de ces résultats, l'équation (1) s'écrit (pour la composante dans la direction $x$ )

$$
\begin{aligned}
I_{x}=\frac{B}{2 \omega} \operatorname{Re}\{ & k^{3} \sum_{m, n} v_{m} v_{n}^{*} \cos \alpha_{m}+k^{3} \sum_{m, n} v_{m} v_{n}^{*} \cos ^{2} \alpha_{m} \cos \alpha_{n} \\
& \left.+\nu k^{3} \sum_{m, n} v_{m} v_{n}^{*} \sin ^{2} \alpha_{m} \cos \alpha_{n}+(1-\nu) k^{3} \sum_{m, n} v_{m} v_{n}^{*} \cos \alpha_{m} \sin \alpha_{m} \sin \alpha_{n}\right\}
\end{aligned}
$$

Cette expression fait apparaître des produits d'indices $m$ et $n$ qu'il est intéressant de regrouper en termes dus à chaque onde d'indice $m$ et en termes croisés résultats d'interférences :

$$
\begin{aligned}
I_{x}=\frac{B k^{3}}{\omega}\left[\sum_{m}\left|v_{m}\right|^{2} \cos \alpha_{m}+\operatorname{Re}\{\right. & \sum_{\substack{m, n \\
n \neq m}} v_{m} v_{n}^{*} \cos \alpha_{m} \cos ^{2} \frac{\alpha_{m}-\alpha_{n}}{2} \\
& \left.\left.+\frac{\nu}{2} \sum_{\substack{m, n \\
n \neq m}} v_{m} v_{n}^{*} \sin \alpha_{m} \sin \left(\alpha_{m}-\alpha_{n}\right)\right\}\right]
\end{aligned}
$$

Cette équation montre que les termes croisés jouent un rôle sensible pour déterminer la valeur de l'intensité vibratoire dans un champ interférentiel créé par un milieux de propagation fini. A contrario, la superposition d'ondes indépendantes se propageant dans une plaque infinie ne présente pas de termes croisés: c'est le modèle proposé par Noiseux pour lequel l'équation (3) se réduit à

$$
I_{x}=\frac{B k^{3}}{\omega} \sum_{m}\left|v_{m}\right|^{2} \cos \alpha_{m} .
$$



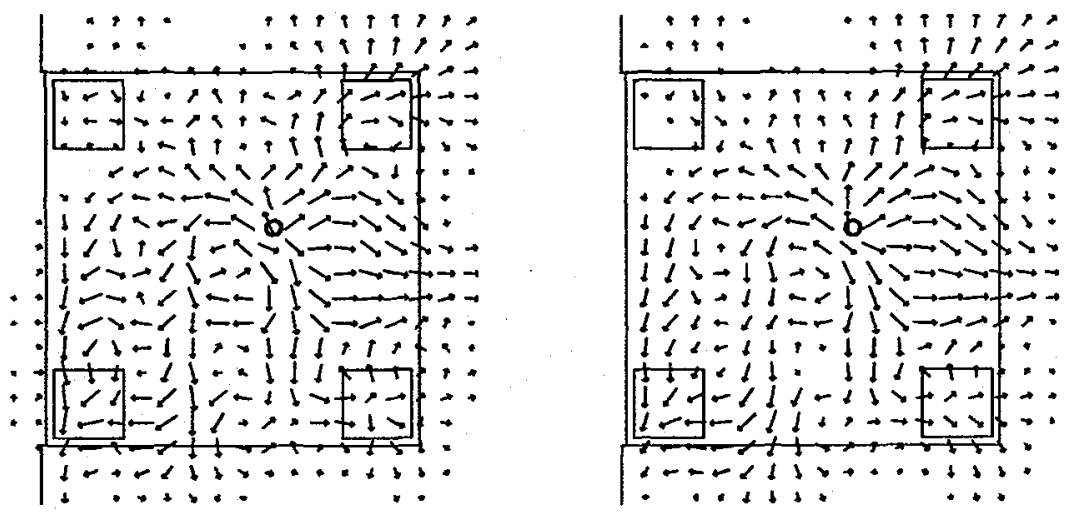

Fig. 1 - Intensité des ondes de flexion déterminée à partir de données expérimentales (o: position du pot vibrant): calcul dans le domaine des nombres d'onde selon (à gauche) la formulation complète de l'éq. (4) et selon (à droite) la formulation approchée de l'éq.(6).

\section{Simplification de la formulation du flux dans les plaques}

Il est montré dans la référence [8] que l'expression de l'intensité des ondes de flexion (1) peut se transformer en une relation plus concise utilisant l'opérateur nabla en trois dimensions, avec $\partial / \partial z \equiv 0$ :

$$
\mathrm{I}=\frac{B}{2 \omega} \operatorname{Im}\left\{\nabla\left(\nabla^{2} v\right) v^{*}-\nabla^{2} v \nabla v^{*}-\frac{1-\nu}{2} \nabla \times \nabla \times\left(v \nabla v^{*}\right)\right\}
$$

Le modèle de champ lointain que décrit l'équation (2) où les ondes planes interferent entre elles permet de satisfaire la relation $\nabla^{2} v=-k^{2} v$, ce qui conduit à une égalité des deux premiers termes de l'expression (4)

$$
\begin{aligned}
\mathbf{I} & \approx \frac{B}{2 \omega} \operatorname{Im}\left\{2 k^{2} v \nabla v^{*}-\frac{1-\nu}{2} \nabla \times \nabla \times\left(v \nabla v^{*}\right)\right\} \\
& \approx \sqrt{B M_{s}} \operatorname{Im}\left\{v \nabla v^{*}-\frac{1-\nu}{4 k^{2}} \nabla \times \nabla \times\left(v \nabla v^{*}\right)\right\} .
\end{aligned}
$$

Cette expression correspond donc à une relation exacte en champ lointain. Si le premier terme peut s'exprimer simplement à partir de différences finies entre 4 points, le second terme nécessite une formulation bien plus complexe. Cependant, ce terme ne met en jeu que des rotationnels et n'existe qu'en fonction des interférences entre ondes planes. Si l'équation (2) représente la superposition d'ondes indépendantes, les phénomènes d'interférence disparaissent et ce terme devient nul. On obtient alors la formulation simplifiée à 4 capteurs pour le champ lointain et le champ libre

$$
\mathrm{I} \approx \sqrt{B M_{3}} \operatorname{Im}\left\{v \nabla v^{*}\right\} .
$$

Elle est équivalente à l'expression de l'intensité des ondes de flexion en champ lointain pour les poutres. Les champs vibratoires sont supposés être constitués par la superposition d'ondes planes indépendantes en propagation libre, et de ce fait, le champ proche des sources et les interférences dues aux réflexions créées par les discontinuités ou les bords d'une plaque ne sont pas pris en compte à priori. Des études de ce type d'approximation $[2,7]$ ont montré les limites de cette approche, mais aussi la surprenante robustesse de l'estimateur (6) en regard de l'importance des approximations (surtout l'hypothèse de champ libre) qui ont permis de le dériver de l'expression (4). C'est ce que montre la figure 1, où l'intensité des ondes de flexion est calculée selon les équations (4) et (6). Les données (expérimentales) sont constituées par le champ de la vitesse complexe normale à la fréquence de $250 \mathrm{~Hz}$ dont la transformation de Fourier spatiale permet 
d'obtenir les différentes dérivées sans approximations [8]. Les grandes tendances du flux sont conservées avec l'expression de champ libre pour laquelle seules les régions présentant des tourbillons sont affectées de façon sensibles (orientation et amplitude des vecteurs). Une explication peut être donnée en considérant que la forme générale de l'intensité des ondes de flexion décrite par l'expression (4) peut être représentée par deux champs potentiels, tels que

$$
\mathbf{I}=-\nabla \phi+\nabla \times \mathbf{C}
$$

Le potentiel vecteur $\mathbf{C}$ produit la composante de l'intensité structurale liée aux phénomènes d'interférence alors que le potentiel scalaire $\phi$ est seul nécessaire pour décrire le bilan du flux de puissance net d'une source vers une zone dissipative ou une sous-structure. Les deux potentiels $\phi$ et $\mathbf{C}$ sont présents dans les deux premiers termes de l'équation (4), mais il est clair que le troisième terme dépend seulement du potentiel vecteur $\mathbf{C}$. De ce fait, il contribue uniquement à la représentation des phénomènes de circulation d'énergie (tourbillons) et son absence dans la formule approchée (6) n'a pas d'impact sur l'évaluation quantitative des transferts d'énergie. Ainsi, si on veut quantifier la puissance injectée dans une plaque en déterminant le flux sur un contour fermé entourant une zone d'excitation, on obtiendra le même résultat en utilisant l'équation (5) et l'équation (6). Cette détermination sera exacte si le contour fermé se trouve dans le champ lointain des excitations mécaniques.

\section{Conclusion}

Une formulation exacte en champ lointain a été dérivée à partir de l'équation exacte du flux des ondes de flexion dans les plaques. Celle-ci présente encore l'inconvénient de nécessiter un nombre de capteurs trop important pour être évaluer simplement par différence finie. Toutefois, l'ajout d'une hypothèse de champ libre permet de la simplifier sensiblement pour aboutir à une formulation pratique à 4 capteurs. Les résultats observés en appliquant cette approche sont meilleurs que ne le laissaient supposer les restrictions imposées par les hypothèses. La formulation avec 4 capteurs décrit assez correctement les cartes de flux (module et orientation des vecteurs) en dehors des régions de tourbillons et conduit à une évaluation quantitative exacte du flux total quand les mesures sont réalisées en champ lointain.

\section{Références}

[1] G. Pavić, "Measurement of structure borne wave intensity, Part I : Formulation of the methods", J. Sound Vib. $49(2), 221-230(1976)$.

[2] M.C. McGary, "Simulated measurement of power flow in structures near to simple sources and simple boundaries", NASA Technical Memorandum 89124, Langley Research Center, Hampton, USA (1988).

[3] D.U. Noiseux, "Measurement of power flow in uniform beams and plates", J. Acoust. Soc. Am. 47 (1), 238-247 (1970).

[4] P. Rasmussen, G. Rasmussen, "Intensity measurements in structures", 11th Int. Cong. Acoust. Proc. 6, Paris, 19-27 July 1983, 231-334.

[5] D. Quinlan, "Adaptation of the four channel technique to the measurement of power flow in structure", Proc. 2nd Int. Cong. on Acoust. Intensity, Senlis, France, 23-26 Sept. 1985, 227-234.

[6] P. Kruppa, "Measurement of structural intensity in building constructions", J. Applied Acoust. 19, 61-74 (1986).

[7] X. Carniel, L. Gavrić, "Etude des formulations de l'intensité vibratoire pour les plaques en flexion", Proc. Cong. on Structural Intensity and Vibrational Energy Flow, Senlis, France, 27-29 Aug. 1990, 323-330.

[8] J.C. Pascal, T. Loyau, J.A. Mann III, "Structural intensity from spatial Fourier transformation and BAHIM acoustical holography method", Proc. Cong. on Structural Intensity and Vibrational Energy Flow, Senlis, France, 27-29 Aug. 1990, 197-204. 